\title{
Nonlocal Four-Point Boundary Value Problem for the Singularly Perturbed Semilinear Differential Equations
}

\author{
Robert Vrabel \\ Institute of Applied Informatics, Automation and Mathematics, Faculty of Materials Science and \\ Technology, Hajdoczyho 1, 91701 Trnava, Slovakia \\ Correspondence should be addressed to Robert Vrabel, robert.vrabel@stuba.sk
}

Received 21 April 2010; Revised 9 September 2010; Accepted 13 September 2010

Academic Editor: Daniel Franco

Copyright (C) 2011 Robert Vrabel. This is an open access article distributed under the Creative Commons Attribution License, which permits unrestricted use, distribution, and reproduction in any medium, provided the original work is properly cited.

This paper deals with the existence and asymptotic behavior of the solutions to the singularly perturbed second-order nonlinear differential equations. For example, feedback control problems, such as the steady states of the thermostats, where the controllers add or remove heat, depending upon the temperature detected by the sensors in other places, can be interpreted with a secondorder ordinary differential equation subject to a nonlocal four-point boundary condition. Singular perturbation problems arise in the heat transfer problems with large Peclet numbers. We show that the solutions of mathematical model, in general, start with fast transient which is the so-called boundary layer phenomenon, and after decay of this transient they remain close to the solution of reduced problem with an arising new fast transient at the end of considered interval. Our analysis relies on the method of lower and upper solutions.

\section{Motivation and Introduction}

We will consider the nonlocal four-point boundary value problem

$$
\begin{aligned}
& \epsilon y^{\prime \prime}+k y=f(t, y), \quad t \in\langle a, b\rangle, k<0,0<\epsilon \ll 1, \\
& y(c)-y(a)=0, \quad y(b)-y(d)=0, a<c \leq d<b .
\end{aligned}
$$

We focus our attention on the existence and asymptotic behavior of the solutions $y_{\epsilon}(t)$ for singularly perturbed boundary value problem (1.1), (1.2) and on an estimate of the difference between $y_{\epsilon}(t)$ and a solution $u(t)$ of the reduced equation $k u=f(t, u)$ when a small parameter $\epsilon$ tends to zero. 
Singularly perturbed systems (SPS) normally occur due to the presence of small "parasitic" parameters, armature inductance in a common model for most DC motors, small time constants, and so forth. The literature on control of nonlinear SPS is extensive, at least starting with the pioneering work of Kokotović et al. nearly 30 years ago [1] and continuing to the present including authors such as Artstein [2,3], Gaitsgory et al. [4-6].

Such boundary value problems can also arise in the study of the steady-states of a heated bar with the thermostats, where the controllers at $t=a$ and $t=b$ maintain a temperature according to the temperature registered by the sensors at $t=c$ and $t=d$, respectively. In this case, we consider a uniform bar of length $b-a$ with nonuniform temperature lying on the $t$-axis from $t=a$ to $t=b$. The parameter $\epsilon$ represents the thermal diffusivity. Thus, the singular perturbation problems are of common occurrence in modeling the heat-transport problems with large Peclet number [7].

We show that the solutions of (1.1), (1.2), in general, start with fast transient $\left(\left|y_{\epsilon}^{\prime}(a)\right| \rightarrow\right.$ $\infty)$ of $y_{\epsilon}(t)$ from $y_{\epsilon}(a)$ to $u(t)$, which is the so-called boundary layer phenomenon, and after decay of this transient they remain close to $u(t)$ with an arising new fast transient of $y_{\epsilon}(t)$ from $u(t)$ to $y_{\epsilon}(b)\left(\left|y_{\epsilon}^{\prime}(b)\right| \rightarrow \infty\right)$. Boundary thermal layers are formed due to the nonuniform convergence of the exact solution $y_{e}$ to the solution $u$ of a reduced problem in the neighborhood of the ends $a$ and $b$ of the bar.

The differential equations of the form (1.1) have also been discussed in [8] but with the boundary conditions $y^{\prime}(a)=0, y(b)-y(c)=0$, that is, with free end $y_{\epsilon}(a)$. Moreover, we show that the convergence rate of solutions $y_{e}$ toward the solution $u$ of a reduced problem is at least $O(\epsilon)$ on every compact subset of $(a, b)$ (in [8], the rate of convergence is only of the order $O(\sqrt{\epsilon}))$. We will write $s(\epsilon)=O(r(\epsilon))$ when $0<\lim _{\epsilon \rightarrow 0^{+}}|s(\epsilon) / r(\epsilon)|<\infty$.

The situation in the case of nonlocal boundary value problem is complicated by the fact that there are the inner points in the boundary conditions, in contrast to the "standard" boundary conditions as the Dirichlet problem, Neumann problem, Robin problem, periodic boundary value problem [9-12], for example. In the problem considered; there is not positive solution $\widetilde{v}_{\epsilon}$ of differential equation $\epsilon y^{\prime \prime}-m y=0, m>0,0<\epsilon$ (i.e., $\widetilde{v}_{\epsilon}$ is convex) such that $\tilde{v}_{\epsilon}(c)-\tilde{v}_{\epsilon}(a)=u(c)-u(a)>0$ and $\tilde{v}_{\epsilon}(t) \rightarrow 0^{+}$for $t \in(a, b\rangle$ and $\epsilon \rightarrow 0^{+}$, which could be used to solve this problem by the method of lower and upper solutions. The application of convex functions is essential for composing the appropriate barrier functions $\alpha, \beta$ for two-endpoint boundary conditions, (see, e.g., [10]). We will define the correction function $v_{\epsilon}^{\text {(corr) }}(t)$ which will allow us to apply the method.

In the past few years the multipoint boundary value problem has received a wide attention (see, e.g., $[13,14])$ and the references therein. For example, Khan [14] have studied a four-point boundary value problem of type $y(c)-v_{1} y(a)=0, y(b)-v_{2} y(d)=0$ where the constants $v_{1}, v_{2}$ are not simultaneously equal to 1 and $\epsilon=1$.

As was said before, we apply the method of lower and upper solutions to prove the existence of a solution for problem (1.1), (1.2) which converges uniformly to the solution $u$ of the reduced problem (i.e., if we let $\epsilon \rightarrow 0^{+}$in (1.1)) on every compact subset of interval $(a, b)$. As usual, we say that $\alpha_{\epsilon} \in C^{2}(\langle a, b\rangle)$ is a lower solution for problem (1.1), (1.2) if $\epsilon \alpha_{\epsilon}^{\prime \prime}(t)+k \alpha_{\epsilon}(t) \geq f\left(t, \alpha_{\epsilon}(t)\right)$ and $\alpha_{\epsilon}(c)-\alpha_{\epsilon}(a)=0, \alpha_{\epsilon}(b)-\alpha_{\epsilon}(d) \leq 0$ for every $t \in\langle a, b\rangle$. An upper solution $\beta_{\epsilon} \in C^{2}(\langle a, b\rangle)$ satisfies $\epsilon \beta_{\epsilon}^{\prime \prime}(t)+k \beta_{\epsilon}(t) \leq f\left(t, \beta_{\epsilon}(t)\right)$ and $\beta_{\epsilon}(c)-\beta_{\epsilon}(a)=0$, $\beta_{\epsilon}(b)-\beta_{\epsilon}(d) \geq 0$ for every $t \in\langle a, b\rangle$.

Lemma 1.1 (see [15]). If $\alpha_{\epsilon}, \beta_{\epsilon}$ are respectively lower and upper solutions for (1.1), (1.2) such that $\alpha_{\epsilon} \leq \beta_{\epsilon}$, then there exists solution $y_{\epsilon}$ of (1.1), (1.2) with $\alpha_{\epsilon} \leq y_{\epsilon} \leq \beta_{\epsilon}$.

Proof of uniqueness of solution for (1.1), (1.2) will be based on the following lemmas. 
Lemma 1.2 (cf. [16, Theorem 1 (Peano's phenomenon)]). Assume that

(i) the function

$$
h(t, y)=f(t, y)-k y
$$

is nondecreasing with respect to the variable $y$ for each $t \in\langle a, b\rangle$,

(ii) $h \in C(\langle a, b\rangle \times R)$.

If $x_{\epsilon}, y_{\epsilon}$ are two solutions of (1.1), (1.2), then

(a) $x_{\epsilon}(t)-y_{\epsilon}(t)=\tilde{C}=$ const in $\langle a, b\rangle$,

(b) if $\tilde{C}>0(\tilde{C}<0)$, then for each $C_{1}, 0 \leq C_{1} \leq \tilde{C}\left(0 \geq C_{1} \geq \tilde{C}\right)$ the function $y_{\epsilon}(t)+C_{1}$ is a solution of the problem (1.1), (1.2).

Lemma 1.3. If $h$ satisfies the strengthened condition (i)

$\left(i^{\prime}\right)$ the function $h(t, y)=f(t, y)-k y$ is increasing with respect to the variable $y$ for each $t \in\langle a, b\rangle$,

then there exists at most one solution of (1.1), (1.2).

Proof. Assume to the contrary that $x_{\epsilon}, y_{\epsilon}$ are two solutions of the problem (1.1), (1.2). Lemma 1.2 implies that $y_{\epsilon}=x_{\epsilon}+\tilde{c}$ on $\langle a, b\rangle$ for some constant $\tilde{c} \neq 0$. Thus

$$
0=\epsilon y_{\epsilon}^{\prime \prime}(t)-\epsilon x_{\epsilon}^{\prime \prime}(t)=h\left(t, y_{\epsilon}(t)\right)-h\left(t, x_{\epsilon}(t)\right)=h\left(t, x_{\epsilon}(t)+\tilde{c}\right)-h\left(t, x_{\epsilon}(t)\right) \neq 0 .
$$

This is a contradiction.

The following assumptions will be made throughout the paper.

(A1) For a reduced problem $k y=f(t, y)$, there exists $C^{2}$ function $u$ such that $k u(t)=$ $f(t, u(t))$ on $\langle a, b\rangle$.

Denote $\mathscr{\ell}(u)=\{(t, y)|a \leq t \leq b| y-,u(t) \mid<d(t)\}$, where $d(t)$ is the positive continuous function on $\langle a, b\rangle$ such that

$$
d(t)= \begin{cases}|u(c)-u(a)|+\delta, & \text { for } a \leq t \leq a+\frac{\delta}{2} \\ \delta, & \text { for } a+\delta \leq t \leq b-\delta \\ |u(b)-u(d)|+\delta, & \text { for } b-\frac{\delta}{2} \leq t \leq b\end{cases}
$$

$\delta$ is a small positive constant.

(A2) The function $f \in C^{1}(\mathscr{L}(u))$ satisfies the condition

$$
\left|\frac{\partial f(t, y)}{\partial y}\right| \leq w<-k \quad \text { for every }(t, y) \in \mathscr{\ell}(u)
$$




\section{Main Result}

Theorem 2.1. Under the assumptions (A1) and (A2) there exists $\epsilon_{0}$ such that for every $\epsilon \in\left(0, \epsilon_{0}\right\rangle$ the problem (1.1), (1.2) has in $\mathscr{H}(u)$ a unique solution, $y_{e}$, satisfying the inequality

$$
\begin{aligned}
& -v_{\epsilon}^{(\mathrm{corr})}(t)-\widehat{v}_{\epsilon}(t)-C \epsilon \leq y_{\epsilon}(t)-\left(u(t)+v_{\epsilon}(t)\right) \leq \widehat{v}_{\epsilon}(t)+C \epsilon \quad \text { for } u(c)-u(a) \geq 0, \\
& -\widehat{v}_{\epsilon}(t)-C \epsilon \leq y_{\epsilon}(t)-\left(u(t)+v_{\epsilon}(t)\right) \leq v_{\epsilon}^{(\mathrm{corr})}(t)+\widehat{v}_{\epsilon}(t)+C \epsilon \quad \text { for } u(c)-u(a) \leq 0
\end{aligned}
$$

on $\langle a, b\rangle$ where

$$
\begin{aligned}
& v_{\epsilon}(t)=\frac{u(c)-u(a)}{D} \cdot\left(e^{\sqrt{(m / \epsilon)}(b-t)}-e^{\sqrt{(m / \epsilon)}(t-b)}+e^{\sqrt{(m / e)}(t-d)}-e^{\sqrt{(m / \epsilon)}(d-t)}\right), \\
& \widehat{v}_{\epsilon}(t)=\frac{|u(b)-u(d)|}{D} \cdot\left(e^{\sqrt{(m / \epsilon)}(t-a)}-e^{\sqrt{(m / \epsilon)}(a-t)}+e^{\sqrt{(m / \epsilon)}(c-t)}-e^{\sqrt{(m / \epsilon)}(t-c)}\right), \\
& D=\left(e^{\sqrt{(m / \epsilon)}(b-a)}+e^{\sqrt{(m / \epsilon)}(d-c)}+e^{\sqrt{(m / \epsilon)}(c-b)}+e^{\sqrt{(m / \epsilon)}(a-d)}\right) \\
& -\left(e^{\sqrt{(m / \epsilon)}(a-b)}+e^{\sqrt{(m / \epsilon)}(c-d)}+e^{\sqrt{(m / \epsilon)}(b-c)}+e^{\sqrt{(m / \epsilon)}(d-a)}\right),
\end{aligned}
$$

$m=-k-w, C=(1 / m) \max \left\{\left|u^{\prime \prime}(t)\right| ; t \in\langle a, b\rangle\right\}$ and the positive function

$$
\begin{aligned}
& v_{\epsilon}^{(\text {corr })}(t)=\frac{w|u(c)-u(a)|}{\sqrt{m \epsilon}}
\end{aligned}
$$

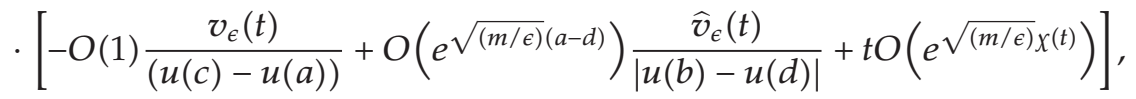

$\chi(t)<0$ for $t \in(a, b\rangle$ and $v_{\epsilon}^{(\text {corr })}(a)=v_{\epsilon}^{(\text {corr })}(c)$.

Remark 2.2. The function $v_{\epsilon}(t)$ satisfies the following:

(1) $\epsilon v_{\epsilon}^{\prime \prime}-m v_{\epsilon}=0$;

(2) $v_{\epsilon}(c)-v_{\epsilon}(a)=-(u(c)-u(a)), v_{\epsilon}(b)-v_{\epsilon}(d)=0$;

(3) $v_{\epsilon}(t) \geq 0(\leq 0)$ is decreasing (increasing) for $a \leq t \leq(b+d) / 2$ and increasing (decreasing) for $(b+d) / 2 \leq t \leq b$ if $u(c)-u(a) \geq 0(\leq 0)$;

(4) $v_{\epsilon}(t)$ converges uniformly to 0 for $\epsilon \rightarrow 0^{+}$on every compact subset of $(a, b\rangle$;

(5) $v_{\epsilon}(t)=(u(c)-u(a)) O\left(e^{\sqrt{(m / \epsilon)} x(t)}\right)$ where $x(t)=a-t$ for $a \leq t \leq(b+d) / 2$ and $x(t)=t-b+a-d$ for $(b+d) / 2<t \leq b$.

The function $\widehat{v}_{\epsilon}(t)$ satisfies the following:

(1) $\epsilon \widehat{v}_{\epsilon}^{\prime \prime}-m \widehat{v}_{\epsilon}=0$;

(2) $\widehat{v}_{\epsilon}(c)-\widehat{v}_{\epsilon}(a)=0, \widehat{v}_{\epsilon}(b)-\widehat{v}_{\epsilon}(d)=|u(b)-u(d)|$;

(3) $\widehat{v}_{\epsilon}(t) \geq 0$ is decreasing for $a \leq t \leq(a+c) / 2$ and increasing for $(a+c) / 2 \leq t \leq b$; 
(4) $\widehat{v}_{\epsilon}(t)$ converges uniformly to 0 for $\epsilon \rightarrow 0^{+}$on every compact subset of $\langle a, b)$;

(5) $\widehat{v}_{\epsilon}(t)=|u(b)-u(d)| O\left(e^{\sqrt{(m / \epsilon)} \hat{x}(t)}\right)$ where $\widehat{X}(t)=c-b+a-t$ for $a \leq t<(a+c) / 2$ and $\widehat{X}(t)=t-b$ for $(a+c) / 2 \leq t \leq b$.

The correction function $v_{\epsilon}^{\text {(corr) }}(t)$ will be determined precisely in the next section.

\section{The Correction Function $v_{\epsilon}^{(\mathrm{corr})}(t)$}

Consider the linear problem

$$
\epsilon y^{\prime \prime}-m y=-2 w\left|v_{\epsilon}(t)\right|, \quad t \in\langle a, b\rangle, \epsilon>0
$$

with the boundary conditions (1.2).

We apply the method of lower and upper solutions. We define

$$
\begin{gathered}
\alpha_{\epsilon}(t)=0 \\
\beta_{\epsilon}(t)=\frac{2 w}{m} \max \left\{\left|v_{\epsilon}(t)\right|, t \in\langle a, b\rangle\right\}=\frac{2 w}{m}\left|v_{\epsilon}(a)\right| .
\end{gathered}
$$

Obviously, $\left|v_{\epsilon}(a)\right|=|u(c)-u(a)|\left(1+O\left(e^{\sqrt{(m / \epsilon)}(a-c)}\right)\right)$ and the constant functions $\alpha, \beta$ satisfy the differential and boundary inequalities required on the lower and upper solutions for (3.1) and the boundary conditions (1.2). Thus on the basis of Lemma 1.1 for every $\epsilon>0$ the unique solution $y_{\epsilon}^{\text {Lin }}$ of linear problem (3.1), (1.2) satisfies

$$
0 \leq y_{\epsilon}^{\operatorname{Lin}}(t) \leq \frac{2 w}{m}|u(c)-u(a)|\left(1+O\left(e^{\sqrt{(m / \epsilon)}(a-c)}\right)\right)
$$

on $\langle a, b\rangle$. The solution we denote by $v_{\epsilon}^{\text {(corr) }}(t)$, that is, the function

$$
v_{e}^{(\text {corr })}(t) \stackrel{\text { def }}{=} y_{e}^{\text {Lin }}(t)
$$

and we compute $v_{\epsilon}^{(\text {corr })}(t)$ exactly as following:

$$
v_{\epsilon}^{(\mathrm{corr})}(t)=-\frac{\left(\psi_{\epsilon}(a)-\psi_{\epsilon}(c)\right)}{(u(c)-u(a))} v_{\epsilon}(t)+\frac{\left(\psi_{\epsilon}(d)-\psi_{\epsilon}(b)\right)}{|u(b)-u(d)|} \widehat{v}_{\epsilon}(t)+\psi_{\epsilon}(t)
$$

where

$$
\psi_{\epsilon}(t)=\frac{w|u(c)-u(a)|}{D \sqrt{m \epsilon}} t\left(e^{\sqrt{(m / \epsilon)}(b-t)}+e^{\sqrt{(m / \epsilon)}(t-b)}-e^{\sqrt{(m / \epsilon)}(d-t)}-e^{\sqrt{(m / \epsilon)}(t-d)}\right)
$$


Hence

$$
\begin{aligned}
\psi_{\epsilon}(a)-\psi_{\epsilon}(c)= & \frac{w|u(c)-u(a)|}{D \sqrt{m \epsilon}} a\left(e^{\sqrt{(m / \epsilon)}(b-a)}+e^{\sqrt{(m / \epsilon)}(a-b)}-e^{\sqrt{(m / \epsilon)}(d-a)}-e^{\sqrt{(m / \epsilon)}(a-d)}\right) \\
& -\frac{w|u(c)-u(a)|}{D \sqrt{m \epsilon}} c\left(e^{\sqrt{(m / \epsilon)}(b-c)}+e^{\sqrt{(m / \epsilon)}(c-b)}-e^{\sqrt{(m / \epsilon)}(d-c)}-e^{\sqrt{(m / \epsilon)}(c-d)}\right) \\
= & \frac{w|u(c)-u(a)|}{\sqrt{m \epsilon}} O(1), \\
\psi_{\epsilon}(d)-\psi_{\epsilon}(b)= & \frac{w|u(c)-u(a)|}{D \sqrt{m \epsilon}} d\left(e^{\sqrt{(m / \epsilon)}(b-d)}+e^{\sqrt{(m / \epsilon)}(d-b)}-2\right) \\
& -\frac{w|u(c)-u(a)|}{D \sqrt{m \epsilon}} b\left(2-e^{\sqrt{(m / \epsilon)}(d-b)}-e^{\sqrt{(m / \epsilon)}(b-d)}\right) \\
= & \frac{w|u(c)-u(a)|}{\sqrt{m \epsilon}} O\left(e^{\sqrt{(m / \epsilon)}(a-d)}\right) \\
\psi_{\epsilon}(t)= & \frac{w|u(c)-u(a)|}{\sqrt{m \epsilon}} t O\left(e^{\sqrt{(m / \epsilon)} x(t)}\right) .
\end{aligned}
$$

Thus, we obtain

$$
\begin{aligned}
v_{\epsilon}^{(\mathrm{corr})}(t)= & \frac{w|u(c)-u(a)|}{\sqrt{m \epsilon}} \\
& \cdot\left[-O(1) \frac{v_{\epsilon}(t)}{(u(c)-u(a))}+O\left(e^{\sqrt{(m / \epsilon)}(a-d)}\right) \frac{\widehat{v}_{\epsilon}(t)}{|u(b)-u(d)|}+t O\left(e^{\sqrt{(m / \epsilon)} x(t)}\right)\right]
\end{aligned}
$$

Hence, taking into consideration (3.8) and the fact that $v_{\epsilon}^{\text {(corr) }}(a)=v_{\epsilon}^{\text {(corr) }}(c)$, the correction function $v_{\epsilon}^{\text {(corr) }}$ converges uniformly to 0 on $\langle a, b\rangle$ for $\epsilon \rightarrow 0^{+}$.

\section{Proof of Theorem 2.1}

First we will consider the case $\mathbf{u}(\mathbf{c})-\mathbf{u}(\mathbf{a}) \geq 0$. We define the lower solutions by

$$
\alpha_{\epsilon}(t)=u(t)+v_{\epsilon}(t)-v_{\epsilon}^{(\text {corr })}(t)-\widehat{v}_{\epsilon}(t)-\Gamma_{\epsilon}
$$

and the upper solutions by

$$
\beta_{\epsilon}(t)=u(t)+v_{\epsilon}(t)+\widehat{v}_{\epsilon}(t)+\Gamma_{\epsilon} .
$$

Here $\Gamma_{\epsilon}=\epsilon \Delta / m$ where $\Delta$ is the constant which shall be defined below, $\alpha \leq \beta$ on $\langle a, b\rangle$ and satisfy the boundary conditions prescribed for the lower and upper solutions of (1.1), (1.2). 
Now we show that $\epsilon \alpha_{\epsilon}^{\prime \prime}(t)+k \alpha_{\epsilon}(t) \geq f\left(t, \alpha_{\epsilon}(t)\right)$ and $\epsilon \beta_{\epsilon}^{\prime \prime}(t)+k \beta_{\epsilon}(t) \leq f\left(t, \beta_{\epsilon}(t)\right)$.

Denote $h(t, y)=f(t, y)-k y$. By the Taylor theorem, we obtain

$$
\begin{aligned}
h\left(t, \alpha_{\epsilon}(t)\right) & =h\left(t, \alpha_{\epsilon}(t)\right)-h(t, u(t)) \\
& =\frac{\partial h\left(t, \theta_{\epsilon}(t)\right)}{\partial y}\left(v_{\epsilon}(t)-v_{\epsilon}^{(\text {corr })}(t)-\widehat{v}_{\epsilon}(t)-\Gamma_{\epsilon}\right),
\end{aligned}
$$

where $\left(t, \theta_{\epsilon}(t)\right)$ is a point between $\left(t, \alpha_{\epsilon}(t)\right)$ and $(t, u(t))$, and $\left(t, \theta_{\epsilon}(t)\right) \in \mathscr{\ell}(u)$ for sufficiently small $\epsilon$. Hence, from the inequalities $m \leq \partial h\left(t, \theta_{\epsilon}(t)\right) / \partial y \leq m+2 w$ in $\mathscr{\ell}(u)$ we have

$$
\begin{aligned}
\epsilon \alpha_{\epsilon}^{\prime \prime}(t)-h\left(t, \alpha_{\epsilon}(t)\right) \geq & \epsilon u^{\prime \prime}(t)+\epsilon v_{\epsilon}^{\prime \prime}(t)-\epsilon v_{\epsilon}^{(\mathrm{corr})^{\prime \prime}}(t)-\epsilon \widehat{v}_{\epsilon}^{\prime \prime}(t)-(m+2 w) v_{\epsilon}(t) \\
& +m v_{\epsilon}^{(\mathrm{corr})}(t)+m \widehat{v}_{\epsilon}(t)+m \Gamma_{\epsilon}
\end{aligned}
$$

Because $v_{\epsilon}(t)=\left|v_{\epsilon}(t)\right|$ we have $-\epsilon v_{\epsilon}^{(\text {corr })^{\prime \prime}}(t)-2 w v_{\epsilon}(t)+m v_{\epsilon}^{(\text {corr })}(t)=0$; as follows from differential equation (3.1), we get

$$
\epsilon \alpha_{\epsilon}^{\prime \prime}(t)-h\left(t, \alpha_{\epsilon}(t)\right) \geq \epsilon u^{\prime \prime}(t)+m \Gamma_{\epsilon} \geq-\epsilon\left|u^{\prime \prime}(t)\right|+\epsilon \Delta
$$

For $\left.\beta_{\epsilon}(t)\right)$ we have the inequality

$$
\begin{aligned}
h\left(t, \beta_{\epsilon}(t)\right)-\epsilon \beta_{\epsilon}^{\prime \prime}(t) & =\frac{\partial h\left(t, \tilde{\theta}_{\epsilon}(t)\right)}{\partial y}\left(v_{\epsilon}(t)+\widehat{v}_{\epsilon}(t)+\Gamma_{\epsilon}\right)-\epsilon \beta_{\epsilon}^{\prime \prime}(t) \\
& =m\left(v_{\epsilon}(t)+\widehat{v}_{\epsilon}(t)+\Gamma_{\epsilon}\right)-\epsilon\left(u^{\prime \prime}(t)+v_{\epsilon}^{\prime \prime}(t)+\widehat{v}_{\epsilon}^{\prime \prime}(t)\right) \\
& \geq \epsilon \Delta-\epsilon\left|u^{\prime \prime}(t)\right|,
\end{aligned}
$$

where $\left(t, \tilde{\theta}_{\epsilon}(t)\right)$ is a point between $(t, u(t))$ and $\left(t, \beta_{\epsilon}(t)\right)$ and $\left(t, \tilde{\theta}_{\epsilon}(t)\right) \in \mathscr{H}(u)$ for sufficiently small $\epsilon$.

The Case: $u(c)-u(a) \leq 0$

The lower solution

$$
\alpha_{\epsilon}(t)=u(t)+v_{\epsilon}(t)-\widehat{v}_{\epsilon}(t)-\Gamma_{\epsilon}
$$

and the upper solution

$$
\beta_{\epsilon}(t)=u(t)+v_{\epsilon}(t)+v_{\epsilon}^{(\text {corr })}(t)+\widehat{v}_{\epsilon}(t)+\Gamma_{\epsilon}
$$


satisfy

$$
\begin{aligned}
\epsilon \alpha_{\epsilon}^{\prime \prime}-h\left(t, \alpha_{\epsilon}\right) & =\epsilon u^{\prime \prime}+\epsilon v_{\epsilon}^{\prime \prime}-\epsilon \widehat{v}_{\epsilon}^{\prime \prime}-\frac{\partial h}{\partial y}\left(v_{\epsilon}-\widehat{v}_{\epsilon}-\Gamma_{\epsilon}\right) \\
& =\epsilon u^{\prime \prime}+\epsilon v_{\epsilon}^{\prime \prime}-\epsilon \widehat{v}_{\epsilon}^{\prime \prime}+\frac{\partial h}{\partial y}\left(-v_{\epsilon}+\widehat{v}_{\epsilon}+\Gamma_{\epsilon}\right) \\
& \geq \epsilon u^{\prime \prime}+\epsilon v_{\epsilon}^{\prime \prime}-\epsilon \widehat{v}_{\epsilon}^{\prime \prime}+m\left(-v_{\epsilon}+\widehat{v}_{\epsilon}+\Gamma_{\epsilon}\right) \\
& =\epsilon u^{\prime \prime}+\epsilon \Delta \geq \epsilon \Delta-\epsilon\left|u^{\prime \prime}\right| \\
h\left(t, \beta_{\epsilon}\right)-\epsilon \beta_{\epsilon}^{\prime \prime} & =\frac{\partial h}{\partial y}\left(v_{\epsilon}+v_{\epsilon}^{(\mathrm{corr})}+\widehat{v}_{\epsilon}+\Gamma_{\epsilon}\right)-\epsilon u^{\prime \prime}-\epsilon v_{\epsilon}^{\prime \prime}-\epsilon v_{\epsilon}^{(\mathrm{corr})^{\prime \prime}}-\epsilon \widehat{v}_{\epsilon}^{\prime \prime} \\
& \geq(m+2 w) v_{\epsilon}+m\left(v_{\epsilon}^{(\mathrm{corr})}+\widehat{v}_{\epsilon}+\Gamma_{\epsilon}\right)-\epsilon u^{\prime \prime}-\epsilon v_{\epsilon}^{\prime \prime}-\epsilon v_{\epsilon}^{(\mathrm{corr})^{\prime \prime}}-\epsilon \widehat{v}_{\epsilon}^{\prime \prime} \\
& =-2 w\left|v_{\epsilon}\right|+m v_{\epsilon}^{(\mathrm{corr})}-\epsilon v_{\epsilon}^{(\mathrm{corr})^{\prime \prime}}+\epsilon \Delta-\epsilon u^{\prime \prime} \\
& =\epsilon \Delta-\epsilon u^{\prime \prime} \geq \epsilon \Delta-\epsilon\left|u^{\prime \prime}\right| .
\end{aligned}
$$

Now, if we choose a constant $\Delta$ such that $\Delta \geq\left|u^{\prime \prime}(t)\right|, t \in\langle a, b\rangle$, then $\epsilon \alpha_{\epsilon}^{\prime \prime}(t) \geq h\left(t, \alpha_{\epsilon}(t)\right)$ and $\epsilon \beta_{\epsilon}^{\prime \prime}(t) \leq h\left(t, \beta_{\epsilon}(t)\right)$ in $\langle a, b\rangle$.

The existence of a solution for (1.1), (1.2) satisfying the above inequality follows from Lemma 1.1 and the uniqueness of solution in $\mathscr{H}(u)$ follows from Lemma 1.3.

Remark 4.1. Theorem 2.1 implies that $y_{\epsilon}(t)=u(t)+O(\epsilon)$ on every compact subset of $(a, b)$ and $\lim _{\epsilon \rightarrow 0^{+}} y_{\epsilon}(a)=u(c), \lim _{\epsilon \rightarrow 0^{+}} y_{\epsilon}(b)=u(d)$. The boundary layer effect occurs at the point $a$ or/and $b$ in the case when $u(a) \neq u(c)$ or/and $u(b) \neq u(d)$.

\section{Acknowledgments}

This research was supported by Slovak Grant Agency, Ministry of Education of Slovak Republic under Grant no. 1/0068/08. The author would like to thank the reviewers for helpful comments on an earlier draft of this article.

\section{References}

[1] P. V. Kokotović, H. K. Khalil, and J. O'Reilly, Singular Perturbation Methods in Control: Analysis and Design, Academic Press, London, UK, 1986.

[2] Z. Artstein, "Stability in the presence of singular perturbations," Nonlinear Analysis: Theory, Methods E Applications, vol. 34, no. 6, pp. 817-827, 1998.

[3] Z. Artstein, "Singularly perturbed ordinary differential equations with nonautonomous fast dynamics," Journal of Dynamics and Differential Equations, vol. 11, no. 2, pp. 297-318, 1999.

[4] Z. Artstein and V. Gaitsgory, "The value function of singularly perturbed control systems," Applied Mathematics and Optimization, vol. 41, no. 3, pp. 425-445, 2000.

[5] V. Gaitsgory, "On a representation of the limit occupational measures set of a control system with applications to singularly perturbed control systems," SIAM Journal on Control and Optimization, vol. 43, no. 1, pp. 325-340, 2004.

[6] V. Gaitsgory and M.-T. Nguyen, "Multiscale singularly perturbed control systems: limit occupational measures sets and averaging," SIAM Journal on Control and Optimization, vol. 41, no. 3, pp. 954-974, 2002. 
[7] A. Khan, I. Khan, T. Aziz, and M. Stojanovic, "A variable-mesh approximation method for singularly perturbed boundary-value problems using cubic spline in tension," International Journal of Computer Mathematics, vol. 81, no. 12, pp. 1513-1518, 2004.

[8] R. Vrábel, "Three point boundary value problem for singularly perturbed semilinear differential equations," Electronic Journal of Qualitative Theory of Differential Equations, vol. 70, pp. 1-4, 2009.

[9] C. De Coster and P. Habets, Two-Point Boundary Value Problems: Lower and Upper Solutions, vol. 205 of Mathematics in Science and Engineering, Elsevier, Amsterdam, The Netherlands, 1st edition, 2006.

[10] K. W. Chang and F. A. Howes, Nonlinear Singular Perturbation Phenomena: Theory and Applications, vol. 56 of Applied Mathematical Sciences, Springer, New York, NY, USA, 1984.

[11] R. Vrábel, "Asymptotic behavior of $T$-periodic solutions of singularly perturbed second-order differential equation," Mathematica Bohemica, vol. 121, no. 1, pp. 73-76, 1996.

[12] R. Vrábel, "Semilinear singular perturbation," Nonlinear Analysis: Theory, Methods E Applications, vol. 25, no. 1, pp. 17-26, 1995.

[13] Y. Guo and W. Ge, "Positive solutions for three-point boundary value problems with dependence on the first order derivative," Journal of Mathematical Analysis and Applications, vol. 290, no. 1, pp. 291-301, 2004.

[14] R. A. Khan, "Positive solutions of four-point singular boundary value problems," Applied Mathematics and Computation, vol. 201, no. 1-2, pp. 762-773, 2008.

[15] J. Mawhin, Points Fixes, Points Critiques et Problèmes aux Limites, Séminaire de Mathématiques Supérieures, no. 92, Presses de l’Université de Montréal, Montreal, Canada, 1985.

[16] V. Šeda, "On some nonlinear boundary value problems for ordinary differential equations," Archivum Mathematicum, vol. 25, no. 4, pp. 207-222, 1989. 